\title{
$B R C A$ genetic testing of individuals from families with low prevalence of cancer: experiences of carriers and implications for population screening
}

\author{
Shiri Shkedi-Rafid, MSc ${ }^{1,2}$, Efrat Gabai-Kapara, MSc ${ }^{3,4}$, Julia Grinshpun-Cohen, MSc ${ }^{4}$ and \\ Ephrat Levy-Lahad, MD ${ }^{3,4}$
}

\begin{abstract}
Purpose: $B R C A$ genes are associated with hereditary breast and ovarian cancers. Guidelines worldwide currently recommend $B R C A$ genetic testing in asymptomatic individuals only if they belong to "high-risk" families. However, population screening for BRCA1/2 may be the logical next step in populations with a high prevalence of founder mutations, such as Ashkenazi Jews. This study aimed to explore (i) the impact of a positive BRCA genetic test result on individuals who have neither a personal history nor a familial history of cancer and (ii) their attitudes toward the concept of population screening.
\end{abstract}

Methods: Semistructured in-depth interviews were carried out with 14 Ashkenazi Jewish women who were asymptomatic BRCA carriers and who belonged to families with low prevalence of cancer.

Results: Three main findings emerged: (i) having no family history of cancer was a source of optimism but also confusion; (ii) engaging in intensified medical surveillance and undergoing preventive procedures was perceived as health-promoting but also tended to induce a sense of physical and psychological vulnerability; and (iii) there was overall support for $B R C A$ population screening, with some reservations.

Conclusion: Women belonging to low-cancer-prevalence families within a "high-risk" ethnic community view BRCA genetic testing positively despite the difficulties entailed, because it allows prevention or early detection of cancer. However, implementing a $B R C A$ population screening program should be carried out with proper pre- and post-testing preparation and support for the individuals undergoing testing.

Genet Med 2012:14(7):688-694

Key Words: Ashkenazi Jews; BRCA; family history; personal experience; population screening

criteria for Ashkenazi Jews with a personal history of breast/ ovarian cancer are less stringent than those for other individuals (for the former, diagnosis at an early age and cancer history in other relatives are not prerequisites for testing). However, the criteria to carry out testing in asymptomatic Ashkenazi Jews are similar to those for other populations, i.e., significant family history of breast/ovarian cancer or a BRCA1/BRCA2 mutation. ${ }^{12}$ Basing risk assessment on positive family cancer history has been demonstrated to have several limitations. Patients may have incorrect information about their family history ${ }^{13}$ and may not bring to medical attention the existence of male relatives with breast cancer. ${ }^{14}$ Health-care providers may not appreciate the importance of paternal family history of cancer. ${ }^{15,16}$ In addition, the family structure could explain low cancer prevalence (e.g., small families or families with preponderance of male individuals). ${ }^{17}$ Therefore, current testing criteria for asymptomatic women may miss potential carriers who are, in fact, at risk. In a study of young patients with breast cancer, $50 \%$ of those who were found to be carriers of $B R C A 1 / B R C A 2$ had no immediate family history of cancer. ${ }^{18}$ Therefore early detection and

\footnotetext{
${ }^{1}$ The Department of Human Genetics, Institute for Medical Research, The Hebrew University of Jerusalem, Jerusalem, Israel; ${ }^{2}$ Centre for Biomedicine and Society, King's College London, London, UK; ${ }^{3}$ Hebrew University Medical School, Jerusalem, Israel; ${ }^{4}$ Medical Genetics Institute, Shaare Zedek Medical Center, Jerusalem, Israel.

Correspondence: Shiri Shkedi-Rafid (Shiri.shkedi@mail.huji.ac.il) 
prevention measures could be offered only with regard to possible future tumors and not for the primary tumor. These issues are particularly pertinent to Ashkenazi Jews, whose recent population history, especially the Holocaust, is reflected in small family size and lack of medical information regarding previous generations in families.

Screening for the three common founder mutations in the general Ashkenazi Jewish population may meet many fundamental screening criteria, as delineated in World Health Organization guidelines ${ }^{19}$ : a severe and common disease (breast/ovarian cancer), a known, identifiable cause (the three BRCA founder mutations), and the availability of preventive measures (e.g., RRSO) or methods of early detection that ameliorate the course of the disease. It is yet to be determined whether Ashkenazi Jews who carry risk-related genes but belong to low-cancer-prevalence families at the population level are sufficiently at risk as to justify population screening. A comparison of cancer risks in carriers from low-prevalence versus high-prevalence families, ascertained through a breast cancer proband, showed similar risks for these two groups: ${ }^{17}$ however, in general, estimates of cancer risk for the general population ${ }^{7}$ have been lower than estimates for those with a personal history of cancer or those belonging to high-risk families. ${ }^{8,9,17}$ In addition, other fundamental issues involving population screening must be considered before disseminating such programs. These issues include the availability and cost-effectiveness of testing, medical surveillance, preventive procedures, counseling, and support for a large number of individuals.

Moreover, population screening for susceptibility genes may have individual and familial ramifications (e.g., potential guilt feelings about passing on the mutation to offspring).$^{20}$ It also raises social, ethical, and legal considerations including privacy and confidentiality; stigmatization; discrimination in insurance and employment; and reproductive issues (e.g., using pregestational/prenatal diagnosis to select against $B R C A$ carriers). ${ }^{21}$

Psychological aspects of $B R C A$ genetic testing have been studied mostly in women with a personal and/or family history of cancer rather than in women with little or no such history, because the latter are not currently being referred for clinical genetic testing. Therefore, results from these studies cannot be generalized to asymptomatic women belonging to low-cancer-prevalence families. This is an important consideration in the context of population screening. The term "lowcancer-prevalence families" is used to indicate families whose history does not fulfill current $B R C A$ testing criteria.

There has been growing interest in various aspects of $B R C A$ population screening. Rubinstein et al. ${ }^{22}$ demonstrated that population $B R C A$ genetic screening of women of the Ashkenazi Jewish population in the United States between the ages of 35 and 55 years would be cost-effective with regard to surgical prevention and treatment of ovarian cancer. Metcalfe et al. ${ }^{23}$ reported a significant response to a newspaper advertisement offering general $B R C A$ genetic testing to Jewish women in Ontario, Canada. Almost half of the carriers identified in their study did not meet current family-history-based testing criteria. Of those who did meet the current testing criteria, $45 \%$ had not been previously referred for genetic testing, demonstrating the existence of health-provider barriers to such testing. In this study, pre- and post-testing questionnaires showed increased post-test distress among those who were found to be carriers ${ }^{24}$ but all the participants expressed a high level of satisfaction with the testing process. No comparisons were made between women belonging to high-prevalence families versus those belonging to low-prevalence families with respect to distress or satisfaction levels. Saunders et al. ${ }^{25}$ reported about an Ashkenazi Jewish family in which the asymptomatic sister of a patient with breast cancer was identified as a carrier through direct-to-consumer testing, even though the affected sister was not a mutation carrier. The authors concluded that current testing criteria are too restrictive with regard to the Ashkenazi Jewish population.

Our study aims to supplement existing knowledge by qualitatively exploring the physical and emotional implications for asymptomatic women belonging to low-cancer-prevalence families on being informed that they are carriers of cancerrelated genetic mutations.

We were especially interested in the motivation for undergoing the test, the everyday consequences of a carrier status, and carriers' attitudes toward $B R C A$ population screening.

\section{MATERIALS AND METHODS}

The participants were asymptomatic women who were carriers of one of the three Ashkenazi Jewish founder mutations and belonged to low-cancer-prevalence families. Most of these participants had been referred for genetic counseling after the finding of a positive test result in a male family member (usually the father), who was tested as part of a study of healthy Ashkenazi males (E. Gabai-Kapara, A. Lahad, B. Kaufman, et al., unpublished data). Asymptomatic carriers belonging to low-cancer-prevalence families were approached if they met the following criteria: $>20$ years of age; underwent the test 1 to 5 years before the current interview; and received pre- and post-testing genetic counseling.

Twenty women met these criteria. They were sent letters explaining the study and inviting them to join, along with a refusal letter that they could return if they were not interested in taking part in the study. If a refusal letter was not received, a genetic counselor from the Medical Genetics Institute at Shaare Zedek Medical Center telephoned the subject to schedule an interview.

Semistructured in-depth interviews were carried out between November 2009 and May 2010. The interviews lasted $1 \mathrm{~h}$ on average, and were audiotaped with the consent of the participants and transcribed verbatim. Analysis was informed by the grounded theory approach. ${ }^{26}$ Whereas in traditional research methods the researcher chooses a theoretical framework, and then applies a model to the studied phenomenon, grounded theory is an inductive approach in which the theory emerges from the data. This approach was thought to be the most suitable for this study, given that this group has not been extensively 
studied and their numbers are currently small. The quoted portions of the interview represent typical responses. The study was approved by the institutional review boards at both Shaare Zedek and Hadassah Medical Centers.

The participants' characteristics are presented in Table 1.

\section{RESULTS}

Of the 20 women approached, accurate contact details were not available for one woman, two sent refusal letters, three refused at the time of the follow-up call, and $14(74 \%)$ consented to participate. The women who consented to participate are believed to be representative of all the women who were approached because the characteristics of women from both groups (detailed in Table 1) were similar. Three themes were particularly prominent in the interviews.

Having no history of cancer in the immediate family was a source of optimism but also of confusion

Unsurprisingly, receiving a positive genetic test result despite having no personal or familial history of cancer came as a shock at first for half of the participants across all ages. More than half of the women (eight) were skeptical when told that their risk of developing cancer was high, because they found the absence of cancer in the immediate family to be a reassuring factor. Three main arguments were advanced as sources of optimism:

1. There are healthy older carriers in the family, who have never had cancer.

"I'm looking at my mother who was a carrier, passed away in her late 80 s and never had cancer... so I'm trying to be optimistic" (62-year-old married woman, mother of three, no family history of breast/ovarian cancer, a brother is a carrier).

2. There has not been much research on carriers who have no personal or family history of cancer.

"I feel as if there are not sufficient data about women like me. I'm sure that in time there will be explanations as to why people have the gene but don't develop the disease" (39-year-old married woman, mother of two, no family history of breast/ovarian cancer, father is a carrier).

3. There may be some protective factor that has yet to be identified.

"We may be protected. Maybe we have some sort of immunity from these cancers" (41-year-old married woman, mother of four, no family history of breast/ ovarian cancer, father is a carrier).

It was particularly hard for these women to handle the mental conflict between their innate optimism and self-assurance on the one hand and the strict medical recommendations on the other. This was especially difficult when irreversible decisions
Table 1 Characteristics of participants at the time of the study

\begin{tabular}{lc} 
Characteristic & $\begin{array}{c}\text { Participants, } \\
\boldsymbol{n}(\%)\end{array}$ \\
\hline Age & \\
$20-29$ & $2(14)$ \\
$30-39$ & $7(50)$ \\
$40-49$ & $3(21)$ \\
$\geq 50$ & $2(14)$
\end{tabular}

Level of education

College/university graduate

College/university postgraduate

5 (36)

Marital status

Married

$12(86)$

Single

1 (7)

Widow

1 (7)

Children

Yes

No

Pregnant

Religiosity

Nonreligious

$10(71)$

Traditional

Religious

Prophylactic oophorectomy

$\begin{array}{lll}\text { Yes } & \text { Below the age of } 40 \quad 34)\end{array}$

No

Above the age of 40

Below the age of 40

Above the age of 40

$1(7)$

Prophylactic mastectomy

Yes

0

No

$14(100)$

Mutation

BRCA1

185 delAG

BRCA1

5382 InsC

0

$B R C A 2$

6174delT

$10(71)$

Family history (first- to third-degree relatives)

No breast/ovarian cancer

Second-degree breast cancer

First-degree breast cancer above the age of 70

Third-degree ovarian cancer

First family member who was genetically tested

Father

Brother

Uncle 
had to be made, mainly with regard to prophylactic procedures. None of the participants underwent risk-reduction mastectomy (RRM), although the procedure was discussed with all of them, including in the summary letter sent to them. Their reasons for not undergoing RRM included confidence in the efficiency of early detection methods; fear of the physical, emotional, and esthetic implications of the surgery; and the fact that they did not consider themselves as being at a sufficiently high risk to warrant such a procedure.

There was less uniformity, however, with regard to the response to the option of RRSO. Although the women acknowledged the lack of efficient surveillance for the early detection of ovarian cancer, the consequence of the procedure, namely, early onset of menopause (given that most participants were premenopausal) meant that it was not an easy decision. In total, four women (37, 40, 49, and 62 years of age) opted to undergo RRSO after a positive BRCA test result, and one other woman had already undergone oophorectomy for other reasons. The majority of the women (nine) were still unsure of the appropriate timing for RRSO, because they were not yet sure whether they had reached their desired family size, because they were not yet married/had no children, or because they feared the side effects of early menopause. One of these women was $>40$ years of age (RRSO is generally medically recommended for high-risk women who are $>40$ years of age).

All the women in the study shifted from engaging in little or no medical surveillance to having close medical surveillance and either undergoing or debating the need for undergoing prophylactic surgeries. The increased focus on continuing medical surveillance in the context of their new increased-cancer-risk status has implications for participants' notions of health, as will be explored next.

\section{Between health and illness}

The genetic test was described by half of the participants as having opened their eyes to the importance of medical surveillance.

Nonetheless, the various tests for the early detection of breast and ovarian cancer (such as magnetic resonance imaging, breast and vaginal ultrasound tests, mammogram, and CA-125 testing) were perceived by many of the participants as being physically burdensome and unpleasant.

This "twilight zone between health and sickness" 27 was even more profound for those having to cope with the physical side effects of early menopause consequent to undergoing the RRSO procedure. Whereas surgery after a diagnosis of disease is performed to achieve cure, a preventive operation that is not instigated by symptoms may render a healthy woman sick, at least temporarily.

From an emotional point of view, a few women voiced a greater sense of vulnerability. In describing the impact of their "carrier status" on their everyday lives, women used expressions such as "constant clouding," "being pursued," "suffocation," a "constant shadow," a "ticking time-bomb," an "axe being lifted above my head," and a "heavy burden."
Yet it seems that, for the majority of the participants, the surveillance tests and their consequences did not disrupt their everyday functioning.

Of note, an understanding of the various physical and emotional implications of being a carrier evolves over time, as these women engage in surveillance tests and face decisions relating to their genetic status. In addition, although most of the women expressed a personal motivation for undergoing genetic testing, a few admitted that they were encouraged to do so by either a parent or a physician. This may lessen the initial understanding of the test's implications, as can be illustrated in this account of the experiences of two sisters:

"We [my sister and I] didn't really know what we were doing [while undergoing genetic testing]; it was mainly to satisfy my father ... I only explored deeply into it, and read about it, when we were specifically told that we were carriers" (41-year-old married woman, mother of four, no family history of breast/ovarian cancer, father is a carrier).

Overall, all the women taking part in this study expressed a strong belief in the benefits of knowledge, as part of the advantages of modern medicine, even where such knowledge resulted in worry. This could also be deduced from their communications with others. Five of the women from our study acted as active advocates for the genetic test, offering it to other women who, in their view, were potential carriers (because of a family history of cancer), so that the other women could also benefit from early detection and prevention. This behavior strengthens the impression that, from the point of view of these women, the benefits of being aware of their genetic status outweighed the drawbacks of having this information.

However, the women's opinions regarding offering the test to women in the general Ashkenazi Jewish population (as distinct from women belonging to "high-risk" families alone) were not necessarily supportive of the idea.

\section{Attitudes toward population screening}

When asked about their own motivation for undergoing genetic testing, the women highlighted the advantages of knowledge, calling it a "blessing," an "asset," and "providence." Not wanting to know, they said, was like "burying your head in the sand," and knowledge was preferable to uncertainty. None of the women interviewed expressed regret about having found out their genetic status or a preference for remaining ignorant of it. On the contrary, they considered themselves lucky for having found it in time rather than too late. A few women even expressed a sense of gratitude toward the first person in the family who had undergone genetic testing.

On this basis, one would expect them to employ similar reasoning regarding the idea of offering the test on a population basis to women without known family histories of cancer. Indeed, there is a general concordance between women's opinions about population screening and their own motivations for being tested. Supporters of population screening considered it to 
be beneficial, acknowledging the importance of genetic knowledge and raising women's awareness, especially when there is no family history of breast and/or ovarian cancer. $B R C A$ testing was even compared to other screening tests offered to women before and during pregnancy, emphasizing the test's relevance to all women, and the high prevalence of the mutations in the Ashkenazi Jewish population.

One respondent said:

"I think that population screening should be offered, just like any other test. For example, all [pregnant] women do the triple test. So why not do this one? I was given a recommendation to have amniocentesis because I had a risk of 1:144 [in my triple-test]. Here we are talking about a 2.5:100 risk [BRCA mutation prevalence]" (41-yearold married woman, mother of four, no family history of breast/ovarian cancer, father is a carrier).

The women emphasized the importance of pre- and posttest counseling to ensure that testing would not be coercive and that women would be well prepared for the implications of a positive test result. A few of the women felt that the information they had received was not sufficiently detailed regarding the postoophorectomy side effects of early menopause. In their words, they wanted to know whether they would have hot flashes, and about possible effects on their sex life or their moods, and so forth. Although they admitted that these details would have not changed their minds regarding the surgery, they felt that it would have made coping with its consequences easier. Therefore, they suggested that first-hand experiences of women undergoing prophylactic surgeries should be included in the counseling session.

However, five of the women were more hesitant about advocating $B R C A$ population screening. They presented six main arguments against $B R C A$ population screening: potential public hysteria; anxiety among certain women; difficulty in drawing a line, as so many other conditions could be potentially tested for as well; problems in having this knowledge before marriage; coercion by physicians to perform the test even if women are undecided; and perception of screening as discriminatory against non-Ashkenazim women. While the usual concern is discrimination against ethnic groups harboring mutations, in Israel, against the backdrop of the historical socioeconomic gap between Ashkenazim and non-Ashkenazim, ${ }^{28}$ preferential testing of Ashkenazim could be perceived as another example of greater opportunities being offered to this ethnic group.

\section{DISCUSSION}

This study aimed to explore the issues that might face asymptomatic individuals belonging to low-cancer-prevalence families if general population screening is initiated for groups with high prevalence of founder mutations.

Asymptomatic individuals with a substantial family history of cancer may begin preparing themselves for a positive test result even before the test is carried out. However, receiving a positive genetic test result could be entirely unexpected for individuals who lack such family history. For them, being "at high risk" is solely a consequence of being identified as a carrier. Albeit not entirely comparable, such an experience has been described with regard to asymptomatic carriers of Fragile $\mathrm{X}$ syndrome. The implications of being a carrier for Fragile $\mathrm{X}$ are independent of the partner's status (with respect to the risk for mental retardation in offspring) and include risks for the woman herself (premature ovarian failure and Fragile X Tremor Ataxia syndrome). Anido et al. found that Fragile X carriers who were identified through participating in a study rather than because of a positive family history were utterly unprepared for the positive results. ${ }^{29}$

Previous studies that examined the experiences of asymptomatic BRCA carriers from high-risk families showed that women were generally willing to accept the immediate physical and mental costs of their preventive actions if these provided a way to avoid future illness. ${ }^{27,30-32}$ The results of our study demonstrate that even women with no family history of cancer are willing to undergo physical and mental discomfort to avoid potential future illness. It could be suggested that the combination of the fear of illness and belief in the ability of technology to ameliorate risks drives women to accept difficulties as long as they are provided with hope.

All the women in the study adhered to surveillance measures. Regarding preventive surgery, three of the four women $\geq 40$ years of age underwent RRSO; this is comparable with the $67 \%$ acceptance of RRSO by BRCA carriers in Israel belonging to high-cancer-prevalence families. ${ }^{33}$ None of the women in our study underwent RRM, although this measure was discussed. Internationally, there is a wide variation in the rate of acceptance of RRM; in Israel only $4.2 \%$ of $B R C A$ carriers from high-cancer-prevalence families undergo RRM. ${ }^{33}$ Therefore the participants' decisions regarding this procedure most probably reflect their cultural context as well, and not merely their personal feelings.

Looking at women's personal experiences of being carriers has several potential implications for the implementation of a $B R C A$ population screening program. The fact that some of the participants compared BRCA testing with screening tests carried out before and during pregnancy may hint at the acceptance of such a screening program in Israel. In Israel, couples planning a pregnancy commonly undergo screening for carrier status for various recessively inherited conditions, based on their ethnic origin. ${ }^{34} \mathrm{~A}$ high acceptance of such tests has been demonstrated particularly in the Ashkenazi Jewish population, which is offered the largest range of tests. ${ }^{35}$ Testing for carrier status is often considered a "must" in Israel, and women who choose not to be tested face criticism from their families. ${ }^{36}$ In light of the "routinization" of prenatal testing ${ }^{37}$ and the comparisons made by a few of the participants between $B R C A$ testing and prenatal testing, attention should be given to avoiding such routinization while implementing $B R C A$ population screening. This is of added importance given that many of the women specifically mentioned that they had undergone genetic testing only to satisfy their relatives and that, initially, 
the implications of having a positive carrier status had not been thoroughly thought out or understood. It is important that population screening, if implemented, occurs as an opt-in rather than an opt-out process, one that allows women to make an informed decision.

The importance of pre- and post-test genetic counseling was expressed in the women's accounts and in various guidelines. ${ }^{38,39}$ Because the increasing use of genetic tests may render pretest counseling impractical (given the constraints of cost, time, and availability of experienced teams), one possibility is to limit posttest counseling only to carriers. In the Ontario study, ${ }^{24}$ women had genetic testing after receiving only written information, whereas in-person genetic counseling was provided only to carriers after the test. More than half of the carriers and 18\% of the noncarriers stated that they would have preferred pretest genetic counseling. One-fifth of the noncarriers stated that they would have preferred to receive their (negative) test result in person rather than by phone or mail. In the realm of $B R C A$ population screening, pre- and post-testing genetic counseling for all women undergoing tests may be costly and therefore unrealistic, suggesting the need to produce an alternative solution. Moreover, the results of our study, in which women did receive pre- and post-testing genetic counseling, indicate that a proper understanding of the implications of a positive carrier status is a gradual process. Therefore, posttest support should be provided to carriers, especially to those who express a greater sense of vulnerability or have difficulties in adjusting to the consequences of preventive procedures.

None of the women in our study opted for RRM, and none of those who underwent RRSO regretted doing so. However, the uncertainty expressed by some of the women regarding the appropriate timing of RRSO, and the perception of coercion with respect to having genetic testing voiced by a few of the women, warrant consideration. Careful attention must be given to evaluate women's preparedness to undergo genetic testing as well as irreversible preventive surgeries, even if such procedures are medically recommended.

This study has several limitations: in spite of a fairly high response rate $(74 \%)$, women with no personal or family history of cancer are not currently referred for BRCA genetic testing, and therefore this study is based on a relatively small sample size. The participants who came forward for genetic testing already knew that they had a 50\% chance of being carriers (because a parent or a sibling was a carrier). In a population screening context, some women will arrive at the point of genetic testing with similar knowledge, whereas others will be the first in their families to be tested. In addition, in view of the fact that only one of the participants in our study was unmarried and childless, the concerns that would confront younger, unmarried, and childless women require further examination.

The study was retrospective, and therefore beyond recall bias, participants in this study (as with participants in comparable studies) may represent those who coped best, leading to biased ascertainment of the number of women with a favorable response to testing. Women with difficulties in coping with their positive test result and its consequences may be more reluctant to discuss the process and their voices would be lost. Another initial source of possible bias is that participants were selected from those who chose to be tested in the first place, and women who initially refused testing may have completely different attitudes and beliefs. Furthermore, all the participants were recruited from a single genetics clinic in Israel. This has several implications: Israel has a national health insurance for all citizens, and there is complete coverage for screening and prevention measures recommended to carriers of risk-related genes. Therefore, the women in our study voiced no concerns regarding access to care. The expenses involved in undergoing the recommended tests (e.g., breast magnetic resonance imaging) and surgical procedures could be a significant issue for carriers in countries that lack full health coverage. In Israel technology is highly valued and therefore new tests are rapidly endorsed, with often little discussion of their medical, social, and ethical ramifications. ${ }^{40}$ Consequently, the experiences of the participants in our study may not be representative of those of women in other countries with different health systems, views, and cultural backgrounds. In addition, all the participants in our study were well educated. Therefore, although they were perhaps representative of women who are likely to undergo screening, they are not representative of the entire population. The conclusions drawn may therefore not be readily applicable to less-educated individuals.

The results of this study show an overall positive experience with $B R C A$ testing and subsequent follow-up procedures, and positive attitudes toward an opt-in $B R C A$ population screening program from a sample of women representing the target population. However, before implementing such screening programs, it is desirable to undertake further research with larger numbers of individuals belonging to low-cancerprevalence families, of different ages and cultural settings, to promote a better understanding of various issues relating to population screening, including medical, psychological (both pre- and posttest assessments), reproductional, and marital issues.

\section{ACKNOWLEDGMENTS}

This study was supported in part by the Breast Cancer Research Foundation (BCRF, NY; to E.L.-L.), the Israel National Institute for Health Policy Research (grant no. 03/54/A, to E.L.-L.), the AngloIsrael Association, and the Leo Baeck (London) Lodge B'nai B'rith (to S.S.-R.). We thank the participants for their willingness to participate and share their experiences. This study could not have been accomplished without the assistance of all members of the Noga clinic in the Shaare Zedek Medical Center, in particular Pnina Mor. We thank Vardiella Meiner, Barbara Prainsack, Yael Hashiloni-Dolev, Roy Gilbar, and Ilana Lowey for their helpful comments on earlier versions of this article.

\section{DISCLOSURE}

The authors declare no conflict of interest. 


\section{REFERENCES}

1. Whittemore AS, Gong G, John EM, et al. Prevalence of BRCA1 mutation carriers among US non-Hispanic Whites. Cancer Epidemiol Biomarkers Prev 2004;13(12):2078-2083.

2. Whittemore AS, Gong G, Itnyre J. Prevalence and contribution of BRCA1 mutations in breast cancer and ovarian cancer: results from three US population-based case-control studies of ovarian cancer. Am J Hum Genet 1997;60(3):496-504.

3. Ford D, Easton DF, Peto J. Estimates of the gene-frequency of brca1 and its contribution to breast and ovarian-cancer incidence. Am J Hum Genet 1995;57(6): 1457-1462.

4. Antoniou AC, Pharoah PD, McMullan G, et al. A comprehensive model for familial breast cancer incorporating BRCA1, BRCA2 and other genes. Br J Cancer 2002;86:76-83.

5. Phelan CM, Kwan E, Jack E, et al. A low frequency of non-founder BRCA1 mutations in Ashkenazi Jewish breast-ovarian cancer families. Hum Mutat 2002;20:352-357.

6. Roa BB, Boyd AA, Volcik K, Richards CS. Ashkenazi Jewish population frequencies for common mutations in BRCA1 and BRCA2. Nat Genet 1996;14:185-187.

7. Struewing JP, Hartge $P$, Wacholder $S$, et al. The risk of cancer associated with specific mutations of BRCA1 and BRCA2 among Ashkenazi Jews. N Engl J Med 1997;336:1401-1408.

8. Ford D, Easton DF, Bishop DT, Narod SA, Goldgar DE. Risks of cancer in BRCA1-mutation carriers. Breast Cancer Linkage Consortium. Lancet 1994;343:692-695.

9. Antoniou AC, Pharoah PD, Narod S, et al. Breast and ovarian cancer risks to carriers of the BRCA1 5382insC and 185delAG and BRCA2 6174delT mutations: a combined analysis of 22 population based studies. J Med Genet 2005;42:602-603.

10. Chen S, Parmigiani G. Meta-analysis of BRCA1 and BRCA2 penetrance. J Clin Oncol 2007;25:1329

11. Domchek SM, Friebel TM, Singer CF, et al. Association of risk-reducing surgery in BRCA1 or BRCA2 mutation carriers with cancer risk and mortality. JAMA 2010;304:967-975.

12. NCCN. National Comprehensive Cancer Network (NCCN) guidelines. http:// www.ncen.org. 2011

13. Murff HJ, Spigel DR, Syngal S. Does this patient have a family history of cancer? An evidence-based analysis of the accuracy of family cancer history. JAMA 2004;292:1480.

14. Schiffman SC, Chagpar AB. Does a family history of male breast cancer influence risk perception and use of genetic testing? Am Surg 2010;76: 879-882.

15. Yong MC, Zhou XJ, Lee SC. The importance of paternal family history in hereditary breast cancer is underappreciated by health care professionals. Oncology 2003;64(3):220-226.

16. Culver JO, Edwards QT, MacDonald DJ, Weitzel JN. USPSTF guidelines will miss BRCA families due to paternal inheritance or a truncated family tree. Ann Intern Med 2005.

17. King MC, Marks JH, Mandell JB; New York Breast Cancer Study Group. Breast and ovarian cancer risks due to inherited mutations in BRCA1 and BRCA2. Science 2003:302:643.

18. Weitzel JN, Lagos VI, Cullinane CA, et al. Limited family structure and BRCA gene mutation status in single cases of breast cancer. JAMA 2007;297:2587.

19. Wilson JM, Jungner YG. Principles and practice of mass screening for disease. 1968. Bol Oficina Sanit Panam 1968;65(4): 281-393.

20. Lynch HT, Snyder C, Lynch JF, et al. Patient responses to the disclosure of BRCA mutation tests in hereditary breast-ovarian cancer families. Cancer Genet Cytogenet 2006;165:91-97.
21. Godard B, ten Kate L, Evers-Kiebooms G, Aymé S. Population genetic screening programmes: principles, techniques, practices, and policies. Eur $J$ Hum Genet 2003;11(suppl 2):S49-S87.

22. Rubinstein WS, Jiang H, Dellefave L, Rademaker AW. Cost-effectiveness of population-based BRCA1/2 testing and ovarian cancer prevention for Ashkenazi Jews: a call for dialogue. Genet Med 2009;11:629.

23. Metcalfe KA, Poll A, Royer $R$, et al. Screening for founder mutations in BRCA1 and BRCA2 in unselected Jewish women. J Clin Oncol 2010;28:387.

24. Metcalfe KA, Poll A, Llacuachaqui $M$, et al. Patient satisfaction and cancer related distress among unselected Jewish women undergoing genetic testing for BRCA1 and BRCA2. Clin Genet 2010;78(5):411-417.

25. Saunders KH, Nazareth S, Pressman PI. Case report: BRCA in the Ashkenazi population: are current testing guidelines too exclusive? Hered Cancer Clin Pract 2011;9:3.

26. Glaser BG, Strauss AL. Grounded Theory: The Discovery of Grounded Theory. de Gruyter: New York, 1967.

27. Dagan E, Goldblatt H. The twilight zone between health and sickness: a qualitative exploration with asymptomatic BRCA1 and 2 mutation carriers. Women Health 2009;49:263-279.

28. Swirski S, Konor-Attias E. Israel: A Social Report 2003. http://www.adva.org/ uploaded/ISRAEL_2003_ENG.pdf.

29. Anido A, Carlson LM, Sherman SL. Attitudes toward fragile X mutation carrier testing from women identified in a general population survey. J Genet Couns 2007;16:97-104.

30. Hamilton R, Williams JK, Skirton H, Bowers BJ. Living with genetic test results for hereditary breast and ovarian cancer. J Nurs Scholarsh 2009;41:276-283.

31. Meijers-Heijboer EJ, Verhoog LC, Brekelmans CT, et al. Presymptomatic DNA testing and prophylactic surgery in families with a BRCA1 or BRCA2 mutation. Lancet 2000;355:2015-2020.

32. van Oostrom I, Meijers-Heijboer H, Lodder LN, et al. Long-term psychological impact of carrying a BRCA1/2 mutation and prophylactic surgery: a 5-year follow-up study. J Clin Onco/ 2003;21:3867.

33. Metcalfe KA, Birenbaum-Carmeli D, Lubinski J, et al.; Hereditary Breast Cancer Clinical Study Group. International variation in rates of uptake of preventive options in BRCA1 and BRCA2 mutation carriers. Int J Cancer 2008;122:2017-2022.

34. Rosner G, Rosner S, Orr-Urtreger A. Genetic testing in Israel: an overview. Annu Rev Genomics Hum Genet 2009;10:175-192.

35. Mishori Dery A, Carmi R, Shoham Vardi I. Different perceptions and attitudes regarding prenatal testing among service providers and consumers in Israel. Community Genet 2007;10(4):242-251.

36. Remennick $L$. The quest for the perfect baby: why do Israeli women seek prenatal genetic testing? Sociol Health IIIn 2006;28:21-53.

37. Suter SM. The routinization of prenatal testing. Am J Law Med 2002; 28(2-3):233-270.

38. McKinnon WC, Baty BJ, Bennett RL, et al. Predisposition genetic testing for late-onset disorders in adults: a position paper of the National Society of Genetic Counselors. JAMA 1997;278(15):1217.

39. Collins FS, Benjamin LJ, Botstein D, et al.; National Advisory Council for Human Genome Research. Statement on use of DNA testing for presymptomatic identification of cancer risk. JAMA 1994;271(10):785.

40. Prainsack $B$, Firestine $O$. Science for survival: biotechnology regulation in Israel. Sci Public Policy 2006;33(1):33-46. 